\title{
Aplicabilidade das equações de referência brasileiras para o teste de caminhada de 6 minutos em pacientes com câncer de pulmão
}

\author{
Applicability of Brazilian reference equations for the 6-minute walk test in patients \\ with lung cancer
}
Aplicabilidad de las ecuaciones de referencia brasileñas para la prueba de caminata de 6 minutos en pacientes con cáncer de pulmón

Nara Naone Lino de Vasconcelos ${ }^{1}$, Neilane da Silva Martins ${ }^{2}$, Eanes Delgado Barros Pereira 3 , Amanda Souza Araújo Almeida ${ }^{4}$, Rafael Mesquita ${ }^{5}$

\begin{abstract}
RESUMO I O objetivo deste estudo foi verificar o impacto da utilização de diferentes equações de referência brasileiras para a distância percorrida no teste da caminhada de 6 minutos (TC6min) na avaliação da capacidade funcional de exercício em pacientes com câncer de pulmão (CP). Este estudo transversal incluiu 48 pacientes com CP (idade média de $60 \pm 12$ anos). Os participantes foram submetidos à avaliação de características sociodemográficas, clínicas e da sua capacidade funcional de exercício com o TC6min, seguindo recomendações internacionais. Foram analisadas cinco equações. A distância percorrida pelos pacientes (503ะ102 metros) foi relativamente próxima às distâncias previstas pelas equações de referência (82-94\% do previsto), embora estatisticamente inferior ( $p<0,05$ para todas).
\end{abstract}

Descritores | Neoplasias Pulmonares; Valores de Referência; Tolerância ao Exercício.

ABSTRACT | The aim of this study was to verify the impact of using different Brazilian reference equations for the distance covered in the 6-minute walk test (6MWT) in the evaluation of functional exercise capacity in patients with lung cancer (LC). This cross-sectional study included 48 patients with LC (average age of $60 \pm 12$ years-old). The participants underwent an assessment of sociodemographic and clinical characteristics and their functional exercise capacity with the 6MWT, following international recommendations. Five equations were analyzed. The distance covered by the patients $(503 \pm 102$ meters) was relatively close to the distances predicted by the reference equations (82-94\% of the predicted), although statistically lower ( $p<0.05$ for all).

Keywords I Lung Neoplasms; Reference Values; Exercise Tolerance.

RESUMEN | El objetivo de este estudio fue verificar el impacto de diferentes ecuaciones de referencia brasileñas para la distancia recorrida en la prueba de caminata de 6 minutos (PC6min) en la evaluación de la capacidad de ejercicio funcional en pacientes con cáncer de pulmón (CP). Este estudio transversal incluyó a 48 pacientes con CP (promedio de edad de 60ะ12 años). Se evaluó las características sociodemográficas, clínicas y la capacidad funcional de los participantes para ejercitarse en la PC6min según los criterios internacionales. Se analizaron cinco ecuaciones. La distancia recorrida por los pacientes (503ะ102 metros) estuvo relativamente cerca de las distancias predichas por las ecuaciones de referencia (82-94\% del predicho), aunque son estadísticamente menores ( $p<0,05$ para todas).

Palabras clave | Neoplasias Pulmonares; Valores de Referencia; Tolerancia al Ejercicio.

\footnotetext{
IUniversidade Federal do Ceará (UFC) - Fortaleza (CE), Brasil. E-mail: naone810@gmail.com. Orcid: 0000-0002-1740-4146 ¿Universidade Federal do Ceará (UFC) - Fortaleza (CE), Brasil. E-mail: neylanemartins.fisio@gmail.com. Orcid: 0000-0001-6327-4007 ${ }^{3}$ Universidade Federal do Ceará (UFC) - Fortaleza (CE), Brasil. E-mail: eanes@fortalnet.com.br. Orcid: 0000-0002-4414-3164 ${ }^{4}$ Universidade Federal do Ceará (UFC) - Fortaleza (CE), Brasil. E-mail: amandasafisio@gmail.com. Orcid: 0000-0003-0129-8980 5Universidade Federal do Ceará (UFC) - Fortaleza (CE), Brasil. E-mail: rafaelmesquita@ufc.br. Orcid: 0000-0002-8048-3393
} 


\section{INTRODUÇÃO}

O câncer de pulmão $(\mathrm{CP})$ está entre os tipos de câncer com maior morbidade e mortalidade em diversas partes do mundo ${ }^{1,2}$. Devido às alterações causadas pelo câncer, como emagrecimento, sintomas como dispneia e fadiga e às consequências dos tratamentos utilizados, como a cirurgia e/ou quimioterapia, pacientes com $\mathrm{CP}$ costumam apresentar uma condição física e mental bastante comprometidas. No estudo de Granger et al. ${ }^{3}$, os autores observaram que pacientes com $\mathrm{CP}$ de não pequenas células caminhavam menos, apresentando um menor número de passos na vida diária, menor distância percorrida no teste da caminhada de 6 minutos (TC6min), pior força de quadríceps e pior qualidade de vida relacionada à saúde no momento do diagnóstico em comparação a indivíduos saudáveis. Comprometimentos físicos foram notados mesmo após terem sido empregados os principais tratamentos nessa população, como a cirurgia de ressecção pulmonar ou a quimioterapia ${ }^{4}$.

Uma revisão sistemática sobre desfechos de medida em pacientes com $\mathrm{CP}$ de não pequenas células evidenciou que o TC6min é o teste mais utilizado para a avaliação da capacidade funcional de exercício ${ }^{3}$. Este analisa a distância máxima que um indivíduo consegue caminhar no período de seis minutos e permite medir a resposta integrada de todos os sistemas envolvidos durante o exercício (por exemplo, cardiovascular, respiratório e musculoesquelético $)^{5}$.

Para a interpretação do resultado do TC6min, uma estratégia bastante usada é a comparação dos valores obtidos da população de interesse com os valores de referência calculados a partir de equações desenvolvidas de uma amostra de indivíduos saudáveis. No Brasil, diversas equações de referência estão disponíveis na literatura ${ }^{6-9}$, porém com características diferentes, como as variáveis levadas em consideração na equação e os coeficientes de determinação $\left(\mathrm{R}^{2}\right)$. Além disso, resultados divergentes têm sido evidenciados na comparação entre as diferentes equações em outras populações ${ }^{10,11}$.

Diante do exposto, o objetivo do presente estudo foi verificar o impacto da utilização de distintas equações de referência brasileiras para a distância percorrida no TC6min na avaliação da capacidade funcional de exercício em pacientes com CP. Acredita-se haver discordância nos resultados das equações. Tal pesquisa torna-se relevante, pois poderá contribuir para uma avaliação mais acurada da capacidade funcional de exercício desses pacientes, o que poderá permitir uma melhor avaliação do impacto de diferentes intervenções, bem como uma melhor avaliação prognóstica. Por exemplo, caso se observe que uma das equações de referência brasileiras apresente resultados muito divergentes em relação às demais, isso pode estar associado a uma incorreta identificação dos pacientes com baixa capacidade funcional de exercício por essa equação, o que poderá levar ao estabelecimento de um prognóstico impróprio ou à prescrição inadequada de uma terapia (por exemplo, exercício físico).

\section{METODOLOGIA}

\section{Delineamento e participantes do estudo}

Trata-se de um estudo transversal, que constitui uma subanálise de um estudo maior que teve o objetivo de avaliar o impacto da ressecção pulmonar por $\mathrm{CP}$ em marcadores inflamatórios após um mês da cirurgia ${ }^{12}$. Essa pesquisa foi realizada no ambulatório de Cirurgia Torácica do Hospital de Messejana Dr. Carlos Alberto Studart Gomes, no município de Fortaleza (CE), Brasil, no período de julho de 2012 a julho de 2014. Para o presente trabalho, foi utilizada apenas a avaliação préoperatória, e foram incluídos os pacientes com diagnóstico de $\mathrm{CP}$ de não pequenas células, candidatos à cirurgia de ressecção pulmonar e com idade maior ou igual a 18 anos; e excluídos os pacientes com $\mathrm{CP}$ avançado ou com alterações cognitivas ou motoras que impossibilitassem as avaliações. Todos os participantes assinaram o Termo de Consentimento Livre Esclarecido e a pesquisa foi aprovada pelo Comitê de Ética em Pesquisa do Hospital de Messejana com o parecer $n^{\circ}$ 277.681. O artigo foi apresentado conforme as recomendações da iniciativa Strengthening the Reporting of Observational Studies in Epidemiology (STROBE) ${ }^{13}$.

\section{Avaliações}

Realizou-se avaliações de dados sociodemográficos e clínicos (idade, sexo, índice de massa corpórea (IMC), estadiamento do câncer, tabagismo atual e doença pulmonar prévia), função pulmonar (espirometria), qualidade de vida relacionada à saúde (medical outcomes study 36-item short form health survey - SF36), e capacidade funcional de exercício (TC6min). A espirometria foi empreendida de acordo com 
as recomendações nacionais ${ }^{14}$, e para a presente análise, foram utilizados os seguintes parâmetros: volume expiratório forçado no primeiro segundo $\left(\mathrm{VEF}_{1}\right)$, capacidade vital forçada $(\mathrm{CVF})$, e a relação entre ambos ${ }^{15,16 .}$

O SF-36 contém 36 itens que se encontram agrupados em oito dimensões (capacidade funcional, dor, aspectos físicos, aspectos emocionais, aspectos sociais, saúde mental, vitalidade e estado geral de saúde). Os resultados podem ser organizados em um coeficiente físico e em um coeficiente mental sumarizados. Os itens do SF-36 são codificados, agrupados e transformados em uma escala de 0 a 100, que representa um estado geral de saúde pior ou melhor, respectivamente, para cada dimensão ou coeficiente sumarizado. Foi usada a versão traduzida e validada para utilização no Brasil ${ }^{17}$.

O TC6min foi exercido de acordo com o preconizado pela American Thoracic Society ${ }^{18}$. Todos os pacientes foram orientados a caminhar a maior distância possível num percurso de 30 metros, durante o tempo de 6 minutos e sem correr. O teste foi realizado duas vezes, mas o utilizado para análise foi o teste com maior distância. A distância percorrida prevista para o TC6min foi calculada de acordo com as seguintes equações de referência para a população brasileira: Iwama et al. ${ }^{6}$, Dourado et al. ${ }^{7}$, Soares e Pereira ${ }^{8}$ e Britto et al. ${ }^{9}$, as quais, a partir deste ponto, serão descritas somente pelo nome do primeiro autor. O estudo de Britto et al. ${ }^{9}$ gerou duas equações, que serão descritas como Britto 1 e Britto 2. Com base na comparação da distância percorrida pelos pacientes com o limite inferior de normalidade estabelecido a partir de cada equação, os participantes foram classificados em capacidade funcional de exercício reduzida ou preservada.

\section{Análise estatística}

A análise dos dados obtidos foi realizada por meio do programa SPSS 22.0 (IBM SPSS Statistics for Windows, Version 22.0. Armonk, NY: IBM Corp.), e o programa GraphPad Prism ${ }^{\circledR} 7.02$ (GraphPad Software Inc., La Jolla (CA) EUA) foi utilizado para a elaboração dos gráficos. A ocorrência de dados faltosos foi descrita quando presente, e as análises foram produzidas considerando-se apenas os dados disponíveis. O teste de Shapiro-Wilk foi usado como teste de normalidade. Dados numéricos foram apresentados como média \pm desvio padrão, média (intervalo de confiança 95\%) ou mediana (intervalo interquartílico). Dados categóricos foram expressos como frequência absoluta e/ou relativa. O teste qui-quadrado foi aplicado para comparar variáveis categóricas. Já para comparar as variáveis contínuas, foram utilizados os testes $\mathrm{t}$ de Student pareado (ou Wilxocon) ou Anova de medidas repetidas (pós-teste de Tukey). A fim de avaliar a concordância entre as diferentes equações para a classificação de capacidade funcional de exercício reduzida foi empregado o coeficiente de kappa. Adotou-se nível de significância menor que $5 \%(p<0,05)$.

\section{RESULTADOS}

\section{Características da amostra}

Foram incluídos 48 pacientes com diagnóstico de $\mathrm{CP}$ de não pequenas células. $\mathrm{Na}$ Tabela 1 , estão descritas as características desses pacientes. Pode-se observar que a amostra abrange pacientes idosos e que a maior parte foi composta por pacientes do sexo feminino (56\%). A média do IMC foi compatível com sobrepeso. Referente ao estadiamento, prevaleceu o estadiamento Ia ou Ib (52\%), e $23 \%$ relataram doença pulmonar prévia. A dimensão física da qualidade de vida mostrou-se mais comprometida do que a mental.

Tabela 1. Características sociodemográficas e clínicas da amostra $(n=48)$

\begin{tabular}{lcr} 
Característica & N & \multicolumn{1}{c}{ Valor } \\
Idade, anos & 48 & $60 \pm 12$ \\
$\begin{array}{l}\text { Sexo, n (\%) } \\
\text { Masculino }\end{array}$ & 48 & $21(44)$ \\
Feminino & & $27(56)$ \\
IMC, kg/m² & 48 & $25,6 \pm 3,2$ \\
Carga tabágica, anos-maço & 37 & $35 \pm 17$ \\
Tabagismo atual, n (\%) & 48 & $5(10)$ \\
Estadiamento, n (\%) & & \\
la ou lb & 48 & $25(52)$ \\
Ila llb ou Illa & & $11(23)$ \\
Doença pulmonar prévia, n (\%) & 48 & $86 \pm 18$ \\
VEF, \% previsto & 48 & $80(73-86)$ \\
VEF $/$ CVF, \% & 48 & $46 \pm 7$ \\
CFS, \% & 48 & $51(42-62)$ \\
CMS, \% & 48 &
\end{tabular}

Dados expressos em frequência absoluta e relativa, média \pm desvio-padrão, ou mediana (intervalo interquartilico). IMC: índice de massa corporal; VEF; : volume expiratório forçado no primeiro segundo; CVF: capacidade vital forçada; CFS: coeficiente físico sumarizado; CMS: coeficiente mental sumarizado.

$\mathrm{Na}$ Tabela 2, estão descritas as respostas ao TC6min. Identificou-se que a distância média percorrida no teste 
pelo grupo foi de $503 \pm 102 \mathrm{~m}$. Observou-se ainda que houve um aumento considerável e estatisticamente significante da FC ( $<<0,001)$, mas nenhuma mudança na $\mathrm{SpO} 2$ após o teste $(\mathrm{p}=0,10)$. Ocorreu um aumento discreto, mas estatisticamente significante nos sintomas de dispneia e fadiga ( $\mathrm{p}<0,001$ para ambos).

Tabela 2. Respostas ao teste da caminhada de 6 minutos em pacientes com câncer de pulmão $(n=48)$

\begin{tabular}{llr} 
Variável & N & \multicolumn{1}{c}{ Valor } \\
Distância no TC6min, metros & 47 & $503 \pm 102$ \\
FC antes, bpm & 37 & $78 \pm 16$ \\
FC após, bpm & 37 & $111 \pm 22$ \\
Delta FC, bpm & 37 & $34 \pm 21$ \\
SpO2 antes, \% & 37 & $97(95-98)$ \\
SpO2 após, \% & 37 & $97(94-98)$ \\
Borg D antes, pontos & 37 & $0(0-0)$ \\
Borg D após, pontos & 37 & $1(0-2)$ \\
Borg F antes, pontos & 37 & $0(0-0)$ \\
Borg F após, pontos & 37 & $1(0-2)$
\end{tabular}

Dados expressos em média \pm desvio-padrão ou mediana (intervalo interquartílico). TC6min: Teste da caminhada de 6 minutos; FC: frequência cardíaca; SpO2: Saturação periférica de oxigênio Borg D: pontuação para dispneia na escala de Borg modificada; Borg F: pontuação para fadiga na escala de Borg modificada.

\section{Distâncias no TC6min previstas}

A Figura 1 apresenta a comparação entre a distância percorrida pelos pacientes e a prevista pelas equações brasileiras. Pode-se notar que a distância percorrida pelos pacientes foi estatisticamente inferior à prevista por todas as equações $(\mathrm{p}<0,05)$. Além disso, observou-se que a distância prevista por Iwama ${ }^{6}(538 \pm 37$ metros $)$ foi inferior à prevista por Dourado ${ }^{7}(593 \pm 56$ metros); a prevista por Dourado ${ }^{7}$ foi superior às distâncias previstas por Soares ${ }^{8}$ (521 \pm 50 metros), Britto $1^{9}$ (549 \pm 38 metros) e Britto $2^{9}$ (545 \pm 53 metros); e a prevista por Soares ${ }^{8}$ foi inferior às previstas por Britto $1^{9}$. A diferença média (intervalo de confiança 95\%) entre os valores alcançados pelos pacientes e os valores previstos de Iwama ${ }^{6}$, Dourado $^{7}$, Soares ${ }^{8}$, Britto $1^{9}$ e Britto $2^{9}$ foi de: $35(11,58)$ metros; $104(80,128)$ metros; 32 (5, 58) metros; $46(21,70)$ metros; e 58 $(31,84)$ metros, respectivamente.

A distância percorrida pelos pacientes representou $93 \pm 15 \%$ do previsto por Iwama ${ }^{6}$ (estatisticamente maior que Britto 1); $82 \pm 13 \%$ do previsto por Dourado ${ }^{7}$ (menor que todas as demais); $94 \pm 15 \%$ do previsto por Soares ${ }^{8}$ (maior que Britto 1); $91 \pm 15 \%$ do previsto por Britto $1{ }^{9}$; e $89 \pm 15 \%$ do previsto por Britto $2^{9}$.

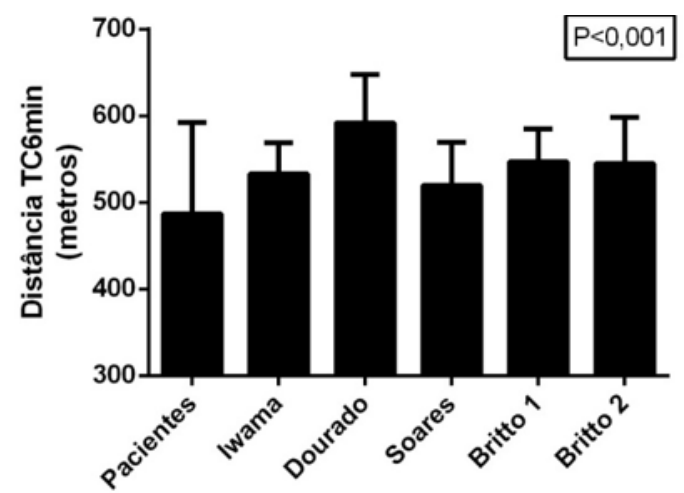

Comparações estatisticamente significantes:
- Pacientes vs.: Todas.
- Iwama vs.: Dourado.
- Dourado vs.: Soares, Britto 1 e Britto 2.
- Soares vs.: Britto 1.

Figura 1. Comparação entre a distância percorrida pelos pacientes com câncer de pulmão e as previstas pelas equações brasileiras ( $n=47$ para Iwama ${ }^{6}$ e Britto 19; $n=35$ para Dourado ${ }^{7}$, Soares ${ }^{8}$ e Britto $2^{9}$ ).

\section{Classificação de acordo com a capacidade funcional de exercício}

A Figura 2 apresenta a proporção de pacientes com $\mathrm{CP}$ classificados como "com baixa capacidade funcional de exercício" segundo as diferentes equações brasileiras. Percebe-se que em Dourado ${ }^{7}$ foi onde a equação apresentou uma maior porcentagem (56\%), seguido por Britto $2^{9}(29 \%)$, Soares ${ }^{8}(19 \%)$, Iwama ${ }^{6}$ e Britto $1^{9}(17 \%$ para ambas). Apenas 7 pacientes (15\%) foram classificados como com capacidade funcional de exercício reduzida por todas as equações, enquanto 20 pacientes (42\%) foram classificados em pelo menos uma das equações. Essa última porcentagem é inferior àquela calculada a partir do estudo de Dourado ${ }^{7}$ devido à ocorrência de dados faltosos (ver legenda da Figura 1).

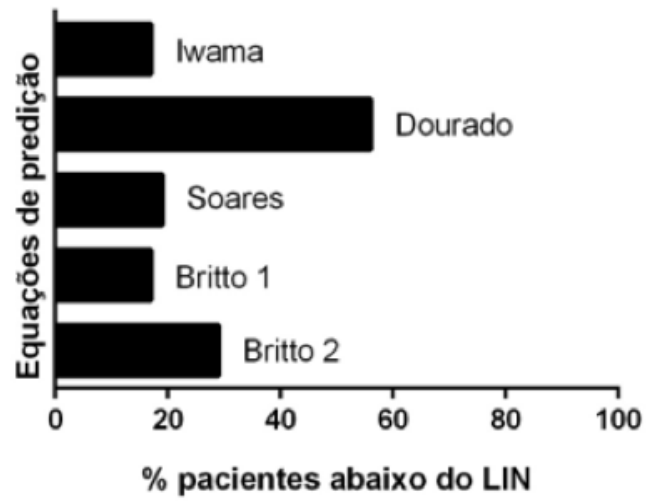

Figura 2. Proporção de pacientes com câncer de pulmão classificados como "com baixa capacidade funcional de exercício" de acordo com as equações brasileiras ( $n=48$ ) 
A concordância pelo coeficiente de kappa entre os pares de equações para a classificação em capacidade funcional de exercício reduzida variou entre 0,32 e 0,92. As equações que apresentaram maior concordância (i.e., kappa $>0,75)$ foram: Iwama ${ }^{6}$ e Soares $^{8}$ : 0,92; Iwama $^{6} \mathrm{e}$ Britto 19: 0,85; Iwama ${ }^{6}$ e Britto 29: 0,85; Soares $^{8}$ e Britto 19: 0,92; e Soares ${ }^{8}$ e Britto $2^{9}: 0,77$.

\section{DISCUSSÃO}

Este estudo investigou a aplicabilidade de diferentes equações de referência brasileiras para a distância percorrida no TC6min em pacientes com CP. Observouse que a distância prevista por Dourado ${ }^{7}$ foi maior que todas as outras, e que não houve diferença na comparação entre as distâncias previstas por Iwama ${ }^{6}$, Soares e Pereira ${ }^{8}$ e uma das equações propostas por Britto ${ }^{9}$. Notou-se ainda uma boa concordância entre as equações previstas por esses últimos estudos para a classificação em capacidade funcional de exercício reduzida. Esses achados podem impactar na interpretação da distância percorrida no TC6min por pacientes com CP.

O principal documento da American Thoracic Society (ATS) e European Respiratory Society (ERS) para a utilização do TC6min em pacientes com doenças respiratórias deixa claro que as equações de referência podem ser usadas para a interpretação dos resultados, mas que elas devem ser verificadas em populações locais sempre que possível ${ }^{5}$. A comparação com valores previstos permite evidenciar o quão limitada uma determinada população é, e essa estratégia tem sido bastante empregada na literatura sobre CP. No estudo de Cavalheri et al. ${ }^{4}$, foi observado que pacientes com $\mathrm{CP}$ de não pequenas células apresentavam uma média de $80 \%$ do previsto para a distância percorrida no TC6min algumas semanas após tratamento curativo. No estudo de Granger et al. ${ }^{3}$, constatou-se uma distância percorrida média de $84 \%$ do previsto no momento do diagnóstico de $\mathrm{CP}$, mas com redução para $69 \%$ do previsto após seis meses. Esses valores são semelhantes ou menores aos encontrados no presente estudo. A identificação de comprometimentos na capacidade funcional de exercício de pacientes com CP é essencial para o desenvolvimento de estratégias de acompanhamento e tratamento a esses indivíduos.

Poucos estudos brasileiros tiveram o objetivo de investigar e comparar a aplicabilidade das diferentes equações para a distância percorrida no TC6min. No estudo de Santos et al. ${ }^{19}$, os autores repararam que a distância percorrida por idosos saudáveis foi inferior àquela prevista por Dourado ${ }^{7}$, superior à prevista por Soares e Pereira ${ }^{8}$ e sem diferença na comparação com os valores de Iwama ${ }^{6}$. No estudo de Machado ${ }^{10}$, os autores investigaram o uso e a aplicabilidade de diferentes equações (nacionais e internacionais) em uma amostra de pacientes com doença pulmonar obstrutiva crônica (DPOC). Foi observado que os valores em porcentagem do previsto calculados a partir das equações de Iwama ${ }^{6} \mathrm{e}$ das duas equações de Britto ${ }^{9}$ foram semelhantes. Notouse também que os valores calculados a partir da equação de Dourado ${ }^{7}$ foram inferiores a todas as demais e que os calculados a partir da equação de Soares ${ }^{8}$ foram superiores a todas as demais. No presente estudo, o valor calculado a partir da equação de Dourado $^{7}$ também foi inferior a todas as demais. No mesmo estudo de Machado et al. ${ }^{10}$, os autores verificaram boa concordância na classificação em capacidade funcional de exercício reduzida entre as equações de Iwama $^{6}$ e as duas equações de Britto ${ }^{9}$. Este trabalho obteve resultados semelhantes, porém não houve boa concordância entre as duas equações propostas por Britto'.

Considerando os achados deste estudo e de estudos prévios, pode-se observar que a equação proposta por Dourado ${ }^{7}$ é a que apresenta resultados mais divergentes das demais. Esse estudo aplicou o TC6min em 98 indivíduos saudáveis e gerou uma equação que incluiu as variáveis idade, peso, altura e sexo que coletivamente explicaram $54 \%$ da variância da distância percorrida no teste. Ainda levando em conta os diferentes estudos, podese afirmar que as equações que apresentam resultados mais semelhantes são a proposta por Iwama $^{6}$ e a equação de Britto $^{9}$, que não utiliza a frequência cardíaca. $\mathrm{O}$ estudo de Iwama ${ }^{6}$ avaliou 134 indivíduos saudáveis e gerou uma equação que incluiu apenas idade e sexo, a qual explicou $30 \%$ da variância na distância percorrida no TC6min. Já o estudo de Britto ${ }^{9}$ incluiu uma amostra multicêntrica de 617 indivíduos saudáveis e gerou duas equações. A equação com resultados semelhantes à proposta por Iwama ${ }^{6}$ incluiu idade, sexo e IMC, explicando 46\% da variância. Essa equação proposta por Britto ${ }^{9}$ e a equação proposta por Iwama ${ }^{6}$ parecem ser as mais aplicáveis para pacientes com CP. A equação proposta por Soares ${ }^{8}$ não foi sugerida com base nos achados de Santos et al. ${ }^{19}$, que perceberam que a mesma levou a valores estatisticamente inferiores aos de uma amostra de indivíduos saudáveis, enquanto a equação Britto $2^{9}$ não foi aconselhada por incluir o delta de frequência cardíaca durante o teste, o que poderia não ser adequado do ponto de vista estatístico. Contudo, para 
uma recomendação mais robusta, são necessários novos estudos que investiguem a capacidade das diferentes equações para discriminar grupos de pacientes com distintas características, a partir da comparação com outros desfechos (p. ex., mortalidade, atividade física na vida diária etc.).

Este estudo apresentou algumas limitações. O tamanho amostral reduzido e a ocorrência de alguns dados faltosos podem ter comprometido o poder de algumas análises, bem como a validade externa dos achados. Além disso, o número de pacientes classificados como "capacidade funcional de exercício reduzida" foi pequeno, o que também pode ter comprometido a validade externa. Todavia, acredita-se que a validade externa não tenha sido tão afetada uma vez que os resultados do TC6min (em porcentagem do previsto) constatados neste estudo para pacientes com CP (i.e., 8294\%) foram próximos aos observados em estudos prévios (i.e., 80-84\%) ${ }^{3,4}$. Por fim, não foram incluídas equações de referência de populações não brasileiras, conforme realizado em outros trabalhos ${ }^{10,11}$. Optou-se por analisar somente as equações brasileiras, considerando que essas são as mais adequadas para serem utilizadas no Brasil.

\section{CONCLUSÕES}

Este estudo observou que $17-56 \%$ dos pacientes com câncer de pulmão têm baixa capacidade funcional de exercício de acordo com as diferentes equações de referência brasileiras. A maioria das equações de referência investigadas exibiu resultados semelhantes. A equação proposta por Dourado et al. ${ }^{7}$ foi a que apresentou resultados mais divergentes, enquanto que as propostas por Iwama et al. ${ }^{6}$ e uma das equações de Britto et al. ${ }^{9}$ (a que não inclui a frequência cardíaca) foram as que levaram a resultados mais semelhantes. Sugere-se que uma dessas duas últimas equações seja utilizada quando pacientes com câncer de pulmão estiverem sendo avaliados.

\section{REFERÊNCIAS}

1. Bray F, Ferlay J, Soerjomataram I, Siegel RL, Torre LA, Jemal A. Global cancer statistics 2018: GLOBOCAN estimates of incidence and mortality worldwide for 36 cancers in 185 countries. CA Cancer J Clin. 2018;68(6):394-424. doi: 10.3322/caac.21492

2. Instituto Nacional de Câncer José Alencar Gomes da Silva (BR). Estimativa 2020: incidência de câncer no Brasil. Rio de Janeiro: Inca; 2019 [cited 2021 Mar 10]. Available from: http://fi-admin. bvsalud.org/document/view/p27p7
3. Granger CL, McDonald CF, Irving L, Clark RA, Gough K, Murnane $A$, et al. Low physical activity levels and functional decline in individuals with lung cancer. Lung Cancer. 2014;83(2):292-9. doi: 10.1016/j.lungcan.2013.11.014

4. Cavalheri V, Jenkins S, Cecins N, Gain K, Phillips M, Sanders LH, et al. Impairments after curative intent treatment for non-small cell lung cancer: A comparison with age and gender-matched healthy controls. Respir Med. 2015;109(10):1332-9. doi: 10.1016/j. rmed.2015.08.015

5. Holland AE, Spruit MA, Troosters T, Puhan MA, Pepin V, Saey $D$, et al. An official European respiratory society/American thoracic society technical standard: Field walking tests in chronic respiratory disease. Eur Respir J. 2014;44(6):1428-46. doi: 10.1183/09031936.00150314

6. Iwama AM, Andrade GN, Shima P, Tanni SE, Godoy I, Dourado VZ. The six-minute walk test and body weight-walk distance product in healthy Brazilian subjects. Braz J Med Biol Res. 2009;42(11):1080-5. doi: 10.1590/S0100-879X2009005000032

7. Dourado VZ, Vidotto MC, Guerra RLF. Equações de referência para os testes de caminhada de campo em adultos saudáveis. J Bras Pneumol. 2011;37(5):607-14. doi: 10.1590/ S1806-37132011000500007

8. Soares MR, Pereira CAC. Teste de caminhada de seis minutos: valores de referência para adultos saudáveis no Brasil. J Bras Pneumol. 2011;37(5):576-83. doi: 10.1590/ S1806-37132011000500003

9. Britto RR, Probst VS, Dornelas de Andrade AF, Samora GAR, Hernandes NA, Marinho PEM, et al. Reference equations for the six-minute walk distance based on a Brazilian multicenter study. Braz J Phys Ther. 2013;17(6):556-63. doi: 10.1590/ S1413-35552012005000122

10. Machado FVC, Bisca GW, Morita AA, Rodrigues A, Probst VS, Furlanetto KC, et al. Agreement of different reference equations to classify patients with COPD as having reduced or preserved 6MWD. Pulmonology. 2018;24(1):16-22. doi: 10.1016/j. rppnen.2017.08.007

11. Marques NLXR, Sá Ferreira A, Silva DPG, Menezes SLS, Guimarães FS, Dias CM. Performance of national and foreign models for predicting the 6-minute walk distance for assessment of functional exercise capacity of Brazilian elderly women. Top Geriatr Rehabil. 2017;33(1):68-75. doi: 10.1097/TGR.0000000000000134

12. Araújo AS, Nogueira IC, Neto AG, Medeiros IL, Morano MTAP, Silva GPF, et al. The impact of lung cancer resection surgery on fibrinogen and C-reactive protein and their relationship with patients outcomes: A prospective follow up study. Cancer Biomark. 2016;16(1):47-53. doi: 10.3233/CBM-150539

13. Von Elm E, Altman DG, Egger M, Pocock SJ, Gøtzsche PC, Vandenbrouckef JP. The Strengthening the Reporting of Observational Studies in Epidemiology (STROBE) Statement: Guidelines for reporting observational studies. Bull World Health Organ. 2007;85(11):867-72. doi: 10.2471/BLT.07.045120

14. Pereira CAC. Espirometria. J Bras Pneumol. 2002;28(Suppl 3):S1-82 [cited 2021 Mar 10]. Available from: http://www.jornaldepneumologia. com.br/detalhe_suplemento.asp?id=45

15. Beck AT, Epstein N, Brown G, Steer RA. An inventory for measuring clinical anxiety: psychometric properties. J Consult Clin Psychol. 1988;56(6):893-7. doi: 10.1037/0022-006X.56.6.893 
16. Gandini RC, Martins MCF, Ribeiro MP, Santos DTG. Inventário de Depressão de Beck - BDI: validação fatorial para mulheres com câncer. Psico-USF. 2007;12(1):23-31. doi: 10.1590/ S1413-82712007000100004

17. Campolina AG, Bortoluzzo AB, Ferraz MB, Ciconelli RM. Validação da versão brasileira do questionário genérico de qualidade de vida short-form 6 dimensions (SF-6D Brasil). Cienc Saude Coletiva. 2011;16(7):3103-10. doi: 10.1590/ S1413-81232011000800010
18. ATS Committee on Proficiency Standards for Clinical Pulmonary Function Laboratories. ATS statement: guidelines for the sixminute walk test. Am J Respir Crit Care Med. 2002;166(1):111-7. doi: 10.1164/rccm.166/1/111

19. Santos LO, Jamami M, Di Lorenzo VAP, Ronchi CF, Arca EA, Pessoa BV. Aplicabilidade das equações de referência para o teste de caminhada de seis minutos em adultos e idosos saudáveis de um município do estado de São Paulo. Fisioter Pesqui. 2013;20(2):172-7. doi: 10.1590/S1809-29502013000200012 